\title{
Impacto de una intervención de educación prenatal en mujeres embarazadas para prevenir el sobrepeso en lactantes
}

\author{
Rosario E. Ortiz-Félix, ${ }^{1}$ Velia M. Cárdenas-Villarreal, ${ }^{2 *}$ Patricia E. Miranda-Félix ${ }^{1}$ y \\ Milton C. Guevara-Valtier

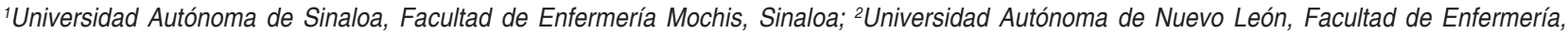 \\ Nuevo León. México
}

\section{Resumen}

Introducción: Durante los primeros 1000 días de vida se establece la base para la salud futura de un niño. Objetivo: Evaluar el impacto de una intervención educativa prenatal en mujeres embarazadas sobre el estado nutricional del hijo desde el nacimiento hasta los cuatro meses de edad. Métodos: Diseño cuasiexperimental de intervención con mujeres a partir de la semana 12 de gestación, asignadas aleatoriamente a un grupo de intervención (GI) para recibir cinco sesiones grupales y tres individuales sobre prácticas de alimentación y percepción materna del peso del hijo y de señales de hambre-saciedad; el grupo control (GC) recibió atención de rutina que incluía al menos tres consultas prenatales. Resultados: 30 mujeres conformaron cada grupo. Después de la intervención, las mujeres del GC practicaron menos lactancia materna exclusiva, fueron propensas a subestimar o sobrestimar el peso del hijo y percibieron con menor intensidad las señales de hambre-saciedad ( $p$ < 0.05). El $80 \%$ de los lactantes del GI presentaron peso normal y $63 \%$ de los niños del GC, una combinación de sobrepeso y obesidad $(p<0.05)$. Conclusiones: El programa de educación prenatal en mujeres embarazadas mostró un efecto significativo en el estado nutricional de los lactantes después de cuatro meses del nacimiento.

PALABRAS CLAVE: Intervención temprana. Lactante. Lactancia materna. Respuesta de saciedad. Mujer embarazada.

\section{Impact of a prenatal education intervention in pregnant women to prevent overweight in infants}

\begin{abstract}
Introduction: During the first 1000 days of life is the basis for a child's future health established. Objective: To evaluate the impact of a prenatal educational intervention in pregnant women on the nutritional status of the child from birth to 4 months of age. Methods: Quasi-experimental intervention design in women with at least 12 weeks of gestation, who were randomly assigned to an intervention group (IG) to participate in five group and three individual sessions on feeding practices and maternal perception of the child's weight and signals of hunger-satiety; the control group (CG) received routine care that included at least three prenatal consultations. Results: Thirty women were included in each group. After the intervention, women in the $C G$ practiced less exclusive breastfeeding, were more likely to underestimate and overestimate the children's weight, and perceived hunger-satiety signals with less intensity $(p<0.05) .80 \%$ of the infants in the IG had normal weight, whereas $63 \%$ of those in the CG had a combination of overweight and obesity $(p<0.05)$. Conclusions: The prenatal education program in pregnant women showed a significant effect on postnatal nutritional status of infants four months after birth.
\end{abstract}

KEY WORDS: Early intervention. Infant. Breastfeeding. Satiety response. Pregnant woman.

Correspondencia:

*Velia M. Cárdenas-Villarreal

E-mail: velia.cardenasvl@uanl.edu.mx
Fecha de recepción: 06-11-2019

Fecha de aceptación: 07-07-2020

DOI: 10.24875/GMM.19005610
Gac Med Mex. 2021;157:3-9

Disponible en PubMed

www.gacetamedicademexico.com

0016-3813/@ 2020 Academia Nacional de Medicina de México, A.C. Publicado por Permanyer. Este es un artículo open access bajo la licencia CC BY-NC-ND (http://creativecommons.org/licenses/by-nc-nd/4.0/). 


\section{Introducción}

La prevalencia de obesidad infantil está aumentando en numerosos países, con graves consecuencias a corto y largo plazo. ${ }^{1}$ México no es la excepción, es más, se ubica en los primeros lugares de prevalencia en el mundo. ${ }^{2}$ Presentar obesidad en la infancia aumenta la probabilidad de continuar con exceso de peso el resto de la vida, ${ }^{3,4}$ de ahí la importancia de prevenirla a temprana edad para combatir esta epidemia.

Estudios recientes recomiendan que las intervenciones preventivas de obesidad infantil deben empezar en los primeros 1000 días de vida, ${ }^{5}$ con el objetivo de orientar y capacitar con un enfoque de bienestar a las futuras madres, para garantizar que sus bebés tengan el mejor comienzo en la vida y que ellas y sus familias logren preservar un peso saludable a lo largo de la vida, así como ayudar a aliviar la carga de las enfermedades no transmisibles en las próximas generaciones.

Informes sobre intervenciones para prevenir obesidad a temprana edad recomiendan que en el periodo perinatal se consideren variables modificables como el control del peso gestacional, la alimentación receptiva, las prácticas de alimentación infantil y la percepción adecuada del peso corporal del hijo. ${ }^{6-8} \mathrm{La}$ alimentación receptiva se refiere a la capacidad de los padres para identificar y responder oportunamente con prácticas adecuadas de alimentación de acuerdo con las señales de hambre y saciedad que emite el niño. ${ }^{6}$ Se ha identificado que las madres con habilidades de alimentación receptiva practican más lactancia materna exclusiva, rutinas de alimentación y evitación del uso de alimentos para calmar a sus hijos, a diferencia de las madres que no tienen esta habilidad. .10 $^{-10}$

En relación con la práctica de alimentación infantil, se sabe que la lactancia materna protege a los niños contra la obesidad y tiene efectos protectores en contra de diversas enfermedades crónicas. ${ }^{6}$ Respecto a la percepción materna del peso del hijo, se ha evidenciado una relación directa con el éxito o fracaso de las medidas preventivas contra la obesidad.,11 Cuando las madres perciben correctamente el peso de sus hijos, son más propensas a elegir alimentos saludables ${ }^{12}$ y a retrasar la introducción de alimentación complementaria. ${ }^{13}$

Cabe resaltar que la mayoría de las intervenciones de prevención de obesidad a temprana edad que han sido analizadas hasta el momento, se han efectuado después del nacimiento del niño y en países de ingresos altos. ${ }^{9-10,14-17}$ Son escasas las intervenciones que han considerado el periodo del embarazo, etapa que da una oportunidad importante a las futuras madres para habilitarlas en la toma de elecciones positivas y comportamientos saludables, tanto durante el embarazo como después del nacimiento del bebé.

Específicamente en México, las intervenciones para prevenir y tratar la obesidad infantil son escasas y se han dirigido a la dieta y el ejercicio, con poco efecto 0 ninguno. ${ }^{18}$ El propósito de este estudio fue evaluar el impacto preliminar de una intervención educativa en mujeres embarazadas enfocada a la percepción materna del peso del hijo, percepción materna de señales de hambre-saciedad y prácticas maternas de alimentación sobre el estado nutricional del hijo posterior a los cuatro meses de nacido.

\section{Métodos}

Se llevó a cabo un estudio cuasiexperimental en el que se reclutaron 80 mujeres gestantes que acudieron a control prenatal en dos instituciones de salud de primer nivel de atención, con tamaños y perfiles demográficos similares en el noreste de México, en el periodo de agosto a octubre de 2018. Fueron excluidas 20 mujeres debido a que no cumplían con los criterios de elegibilidad para el estudio: ser mayores de 18 años, cursar el primer trimestre de embarazo, con embarazo único, sin diagnóstico de complicaciones de enfermedades crónicas. La muestra final estuvo constituida por 60 participantes (Figura 1).

La asignación aleatoria de las mujeres a los grupos de intervención (GI) y control (GC) se realizó mediante un programa computacional y se dio a conocer por escrito a las participantes en un sobre sellado junto con las especificaciones del estudio. El protocolo se apegó a los principios éticos de la Declaración de Helsinki ${ }^{19}$ y fue aprobado por el Comité de Ética en Investigación de la Facultad de Enfermería Mochis, Universidad Autónoma de Sinaloa (CEI-000-35).

El programa de intervención se diseñó con base en la teoría de promoción de la salud de Pender, ${ }^{20}$ que describe que el comportamiento humano es una interacción dinámica y recíproca de factores personales y de habilidades cognitivas y de comportamiento, que determina en las personas la toma de acciones para prevenir problemas de salud. La intervención se integró por tres componentes: educación, reforzamiento 


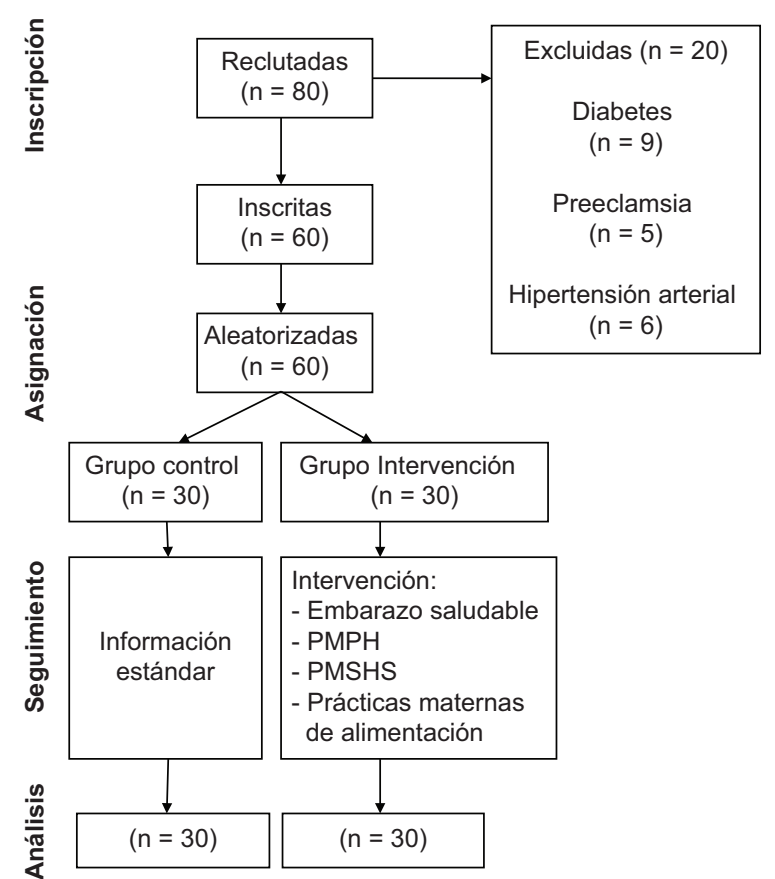

Figura 1. Diagrama de flujo del estudio. $\mathrm{PMPH}=$ percepción materna del peso del hijo; PMSHS = percepción materna de señales de hambre y saciedad.

de habilidades cognitivas y seguimiento posparto (cuatro meses). Las sesiones y contenidos se describen en la Tabla 1. El grupo control recibió la atención prenatal estándar establecida como protocolo en las instituciones de salud participantes (mínimo tres consultas de seguimiento de control prenatal y asesoramiento de dieta y ejercicio).

Se capacitó durante 20 horas presenciales a una enfermera materno-infantil y a un nutriólogo de cada institución de salud, quienes fueron los responsables directos de la entrega de la intervención; así como a dos licenciados en enfermería que colaboraron en la recolección de los datos. Todos utilizaron una guía de facilitador para proporcionar el contenido de cada sesión y para evaluar el proceso de las actividades programadas durante la intervención.

Todas las participantes firmaron el formato de consentimiento informado. Las sesiones grupales se llevaron a cabo con un promedio de ocho a 10 embarazadas en una sala audiovisual y las individuales, en un consultorio de la institución de salud. Se llevaron a cabo recordatorios mediante llamadas telefónicas antes de cada cita de control prenatal.

Antes de la intervención se recolectaron datos sociodemográficos (edad, nivel educativo, estado civil, ingreso económico mensual). Las mediciones
Tabla 1. Contenido de la intervención educativa prenatal para prevenir la obesidad en lactantes

\begin{tabular}{|c|c|c|}
\hline $\begin{array}{l}\text { Tiempo de entrega } \\
\text { (semanas de } \\
\text { gestación) }\end{array}$ & $\begin{array}{l}\text { Sesión } \\
\text { educativa }\end{array}$ & Contenido \\
\hline \multicolumn{3}{|l|}{ Sesiones grupales* } \\
\hline Semanas $12-16$ & 1 & $\begin{array}{l}\text { Estilos de vida saludable en el } \\
\text { embarazo } \\
\text { - Impacto de la obesidad en el } \\
\text { embarazo y en el desarrollo del } \\
\text { feto. } \\
\text { - Monitoreo de aumento de peso } \\
\text { gestacional. }\end{array}$ \\
\hline Semanas 16-20 & 2 & $\begin{array}{l}\text { Prácticas de alimentación } \\
\text { adecuadas } \\
\text { - Lactancia materna exclusiva. } \\
\text { - Técnicas de amamantamiento. } \\
\text { - Métodos de extracción y } \\
\text { conservación de la leche materna. } \\
\text { - Problemas de mamas. }\end{array}$ \\
\hline Semanas 20-24 & 3 & $\begin{array}{l}\text { Alimentación perceptiva y } \\
\text { beneficios } \\
\text { - Reconocimiento de señales } \\
\text { de hambre que emite el niño } \\
\text { durante los primeros meses de } \\
\text { vida (tempranas, activas, tardías) }\end{array}$ \\
\hline Semanas 24-38 & 4 & $\begin{array}{l}\text { Reconocimiento de señales de } \\
\text { saciedad que emite el niño al año } \\
\text { de vida del niño } \\
\text { - Principales señales (tempranas, } \\
\text { activas, tardías). } \\
\text { - Alimentación complementaria. }\end{array}$ \\
\hline Semanas 28-32 & 5 & $\begin{array}{l}\text { Percepción adecuada del peso } \\
\text { de hijo } \\
\text { - Etapas de desarrollo de los } \\
\text { lactantes. } \\
\text { - Importancia del monitoreo del peso. } \\
\text { - Mitos sobre la obesidad-salud } \\
\text { en los lactantes. } \\
\text { - Uso de curvas de crecimiento } \\
\text { de la Organización Mundial de } \\
\text { la Salud. }\end{array}$ \\
\hline
\end{tabular}

Reforzamiento**

Semanas 32-40 Sesión Reforzamiento de habilidades

individual - Demostración de

1 a 3 procedimientos de prácticas de alimentación adecuadas.

- Percepción materna de señales de hambre y saciedad.

- Percepción del peso del hijo.

Seguimiento

Nacimiento hasta los cuatro meses de edad del hijo

Mensajes Motivación para realizar prácticas de texto de alimentación adecuadas telefónico y aclarar dudas sobre su (tres implementación. mensajes)

${ }^{*}$ Concientización de la importancia del control prenatal y habilidades cognitivas para el crecimiento y desarrollo del hijo (60 minutos en cada sesión).

**30 minutos en cada sesión. 
antropométricas (peso y estatura) se tomaron durante el embarazo en la consulta prenatal. El peso pregestacional fue autoinformado por la embarazada; para su evaluación se consideraron los criterios internacionales, ${ }^{21,22}$ respectivamente. Del niño se tomaron los siguientes datos: edad, sexo, peso y talla al nacer.

El resultado primario de este estudio fue el estado nutricional del niño, que se valoró mediante las puntuaciones $Z$ del indicador de crecimiento índice de masa corporal (IMC)/edad, de la Organización Mundial de la Salud, ${ }^{22}$ calculado con el programa Anthro versión 3.2.2. La evaluación se realizó a los cuatro meses de edad del lactante.

La percepción materna de las señales de hambre y saciedad en el lactante se midió mediante las preguntas adaptadas de la Escala de Respuesta a las Señales de Hambre y Saciedad. ${ }^{23}$ Esta herramienta indaga si las madres perciben las señales (hambre 0 saciedad) que comunica un bebé menor de seis meses durante la alimentación y si son tempranas, activas o tardías. Incluye dos ítems: "Si su hijo tiene hambre..." y "Si su hijo está saciado...", seguidas de 10 frases relacionadas con señales de hambre y ocho frases para señales de saciedad. La escala de respuestas es tipo Liker de cinco puntos, donde uno significa nunca y cinco, siempre. La suma de las respuestas se categorizó en baja (18-45), moderada (4666) y alta (67-90). El valor de alfa de Cronbach obtenido de esta escala fue 0.72 .

Para valorar las prácticas de alimentación, se realizaron las siguientes preguntas: "¿Cuánto tiempo dio lactancia materna exclusiva a su hijo?" y "¿A qué edad, su hijo inició alimentación con leche materna de fórmula en biberón?"24 Para medir la percepción materna del peso del lactante se formuló el ítem "Creo que mi hijo está...", con las siguientes opciones de respuesta: bajo peso, un poco de bajo peso, más 0 menos con peso correcto, con un poco de sobrepeso y con sobrepeso. ${ }^{25}$ Se consideró percepción adecuada cuando la respuesta de la madre coincidió con el estado nutricional del hijo al momento de la evaluación. Se consideró subestimar o sobreestimar el peso cuando la madre percibió el peso como menor o mayor al estado nutricional que verdaderamente tenía el hijo, respectivamente.

Los datos se analizaron con el programa estadístico Statistical Package for the Social Sciences (SPSS) versión 20. Se obtuvo estadística descriptiva de las variables sociodemográficas (madre-hijo). Para conocer la normalidad de los datos se aplicó la prueba de Shapiro-Wilk; los datos mostraron distribución no paramétrica. Para las variables cualitativas se utilizó la prueba de chi cuadrada y prueba exacta de Fisher y para las variables cuantitativas, la prueba $U$ de Mann-Whitney; se consideró significación estadística con $p<0.05$.

\section{Resultados}

Al inicio de la intervención no se observaron diferencias significativas en las características sociodemográficas de las mujeres embarazadas, tanto del GI como del GC, con excepción de la ocupación (Tabla 2). Se identificaron diferencias significativas en el IMC posparto de las participantes (26.55 en el GC y 23.95 en el $G I, p=0.043$ ) y en la puntuación $Z$ de IMC/edad de los lactantes a los cuatro meses del nacimiento, que fue mayor en el GC que en el GI $(p=0.034)$.

Al comparar los resultados de las variables de interés a los cuatro meses posparto se observó que las mujeres del Gl practicaron lactancia materna exclusiva en mayor proporción que las del GC (41.70 y $23.30 \%$, respectivamente). Referente a las variables cognitivas, las madres del GC fueron más propensas a subestimar y sobrestimar el peso de sus hijos que las madres del GI (73.30 y $23.30 \%$ ); además, percibieron pobremente las señales de hambre y saciedad (96.70 y $16.70 \%$ ). Finalmente, los lactantes del GI presentaron mayor porcentaje de peso normal $(80.00 \%)$, que los del GC (63.40\%). Todos los resultados mostraron diferencias con significación estadística, $p<0.05$ (Tabla 3). Asimismo, las madres del Gl mostraron un alto porcentaje de alimentación perceptiva $(83.30 \%)$ y mayor realización de lactancia materna exclusiva que las madres del GC $(p=0.009)$, Tabla 4.

\section{Discusión}

En este estudio se identificó que una intervención educativa prenatal en mujeres embarazadas tuvo un impacto sobre el estado nutricional del hijo, al corroborarlo después de los cuatro meses del nacimiento. La orientación sobre los beneficios de la lactancia materna exclusiva, técnicas de extracción de leche materna y conservación de esta, entre otros aspectos, aparentemente influyó en la toma de decisiones de realizar la lactancia materna exclusiva por más tiempo que cuando no se recibió un entrenamiento más extenso sobre estas prácticas, resultado que coincide con los de estudios previos. . $^{9,26,27}$ 
Tabla 2. Características sociodemográficas de madres y lactantes

\begin{tabular}{|c|c|c|c|}
\hline & $\begin{array}{l}\text { Grupo control } \\
\qquad(n=30)\end{array}$ & $\begin{array}{l}\text { Grupo de } \\
\text { intervención } \\
(n=30)\end{array}$ & p \\
\hline \multicolumn{4}{|c|}{ Características demográficas } \\
\hline Edad de la madre & $27.50(20-40)$ & $27.50(19-38)$ & $0.812^{\mathrm{a}}$ \\
\hline Número de hijos & $3(1-5)$ & $2(1-5)$ & $0.179^{a}$ \\
\hline \multicolumn{4}{|l|}{ Estado civil, n (\%) } \\
\hline Con pareja & $25(83.30)$ & $28(93.30)$ & \multirow[t]{2}{*}{$0.212^{c}$} \\
\hline Sin pareja & $5(16.70)$ & $2(6.70)$ & \\
\hline \multicolumn{4}{|l|}{ Escolaridad, $\mathrm{n}(\%)$} \\
\hline Básica & $5(16.70)$ & $4(13.30)$ & \multirow[t]{3}{*}{$0.676^{b}$} \\
\hline Media superior & $17(56.60)$ & $20(66.70)$ & \\
\hline Superior & $8(26.70)$ & $6(20.00)$ & \\
\hline \multicolumn{4}{|l|}{ Ocupación, n (\%) } \\
\hline Hogar & $22(73.30)$ & $14(46.70)$ & \multirow[t]{2}{*}{$0.044^{b}$} \\
\hline $\begin{array}{l}\text { Trabajo } \\
\text { remunerado }\end{array}$ & $8(26.70)$ & $16(53.30)$ & \\
\hline \multicolumn{4}{|c|}{ Sexo del lactante, $n(\%)$} \\
\hline Femenino & $15(50.00)$ & $15(50.00)$ & \multirow[t]{2}{*}{$1.000^{\mathrm{b}}$} \\
\hline Masculino & $15(50.00)$ & $15(50.00)$ & \\
\hline
\end{tabular}

Características antropométricas de la madre

$\begin{array}{llll}\text { Peso } & 69(45.00-85.00) & 65.00(54.00-96.00) & 0.906^{a}\end{array}$ pregestacional $(\mathrm{kg})$

\begin{tabular}{|l|c|c|c|}
\hline GPG $(\mathrm{kg})$ & $9(4-12)$ & $6(4-10)$ & $0.096^{\mathrm{a}}$ \\
\hline Peso actual (kg) & $71.00(56.00-92.00)$ & $66.00(57.00-80.00)$ & $0.030^{\mathrm{a}}$ \\
\hline Talla $(\mathrm{m})$ & $1.64(1.50-1.70)$ & $1.66(1.54-1.77)$ & $0.091^{\mathrm{a}}$ \\
\hline IMC actual & $26.55(19.53-35.00)$ & $23.95(20.80-30.12)$ & $0.043^{\mathrm{a}}$ \\
\hline Estado nutricional, $\mathrm{n}(\%)$ & & \\
\hline Peso normal & $9(30.00)$ & $13(43.30)$ & $0.044^{\mathrm{b}}$ \\
\hline Sobrepeso & $16(53.30)$ & $17(56.70)$ & \\
\hline Obesidad & $5(16.70)$ & $0(\mathrm{~N} / \mathrm{A})$ & \\
\hline
\end{tabular}

Características antropométricas del lactante

Peso al nacer $(\mathrm{kg}) \quad 3.50(2.50-4.00) \quad 3.75(2.50-5.00) \quad 0.100^{\mathrm{a}}$

Talla al nacer (kg) $50.00(45.00-54.00) \quad 50.00(45.00-59.00) \quad 0.737^{a}$

$\begin{array}{llll}\text { Peso actual }(\mathrm{kg}) & 6.45(3.30-9.00) & 5.20(4.20-7.80) & 0.038^{\mathrm{a}}\end{array}$

Talla actual (cm) $62.00(48.00-70.00) 62.00(55.00-66.00) \quad 0.806^{a}$

\begin{tabular}{ll|lll} 
Índice z IMC/edad & $2.04(-2.38-3.85)$ & $0.40(-1.78-2.02)$ & $0.034^{a}$
\end{tabular}

Los valores se expresaron en media (rango) o n (\%).

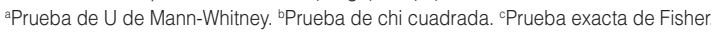

$\mathrm{GPG}=$ ganancia de peso gestacional, IMC = índice de masa corporal.
Tabla 3. Comparación intergrupos de las variables de interés

\begin{tabular}{|c|c|c|c|c|c|}
\hline \multirow[t]{2}{*}{ Variable } & \multicolumn{2}{|c|}{$\begin{array}{l}\text { Grupo } \\
\text { control }\end{array}$} & \multicolumn{2}{|c|}{$\begin{array}{c}\text { Grupo de } \\
\text { intervención }\end{array}$} & \multirow[t]{2}{*}{ p } \\
\hline & n & $\%$ & $\mathrm{n}$ & $\%$ & \\
\hline $\begin{array}{l}\text { Practica de alimentación } \\
\text { Lactancia materna } \\
\text { exclusiva }\end{array}$ & 14 & 23.30 & 25 & 41.70 & $0.003^{b}$ \\
\hline Alimentación con biberón & 16 & 26.70 & 5 & 8.30 & \\
\hline $\begin{array}{l}\text { Percepción materna del } \\
\text { peso del hijo } \\
\text { Adecuada } \\
\text { Subestima/sobrestima }\end{array}$ & $\begin{array}{c}8 \\
22\end{array}$ & $\begin{array}{l}26.70 \\
73.30\end{array}$ & $\begin{array}{c}23 \\
7\end{array}$ & $\begin{array}{l}76.70 \\
23.30\end{array}$ & $0.001^{\mathrm{b}}$ \\
\hline $\begin{array}{l}\text { Percepción señales } \\
\text { hambre/saciedad } \\
\text { Alta } \\
\text { Pobre/moderada }\end{array}$ & $\begin{array}{c}1 \\
29\end{array}$ & $\begin{array}{l}3.30 \\
96.70\end{array}$ & $\begin{array}{c}25 \\
5\end{array}$ & $\begin{array}{l}83.30 \\
16.70\end{array}$ & $0.001^{c}$ \\
\hline $\begin{array}{l}\text { Estado nutricional de los } \\
\text { lactantes } \\
\text { Bajo peso } \\
\text { Normal } \\
\text { Sobrepeso } \\
\text { Obesidad }\end{array}$ & $\begin{array}{c}1 \\
10 \\
11 \\
8\end{array}$ & $\begin{array}{r}3.30 \\
33.30 \\
36.70 \\
26.70\end{array}$ & $\begin{array}{c}0 \\
24 \\
5 \\
1\end{array}$ & $\begin{array}{c}0 \\
80.00 \\
16.70 \\
3.30\end{array}$ & $0.002^{\mathrm{b}}$ \\
\hline
\end{tabular}

Es importante mencionar que la literatura que aborda la percepción del peso del hijo en conjunto con la lactancia materna exclusiva es muy limitada, pero se ha observado que cuando la madre es sensible a percibir el peso de su hijo, elige alimentos más saludables y establece rutinas de alimentación adecuadas. ${ }^{7,12,14}$ En este estudio, las mujeres embarazadas que recibieron asesoría sobre cómo identificar el peso correcto de sus futuros hijos y aclarar mitos sobre el mismo en lactantes fueron más capaces de ponderar la condición nutricional de sus hijos a los cuatro meses de edad. Estos hallazgos sugieren la importancia del acompañamiento y orientación por parte del personal de salud para lograr que las madres valoren adecuadamente el crecimiento y desarrollo de sus hijos desde que estos nacen. ${ }^{13}$

Respecto a la percepción materna de señales de hambre y saciedad, las madres del GI percibieron con mayor intensidad estas y se asociaron a la lactancia materna exclusiva. Lo anterior concuerda con ensayos clínicos aleatorizados ${ }^{8-10}$ que muestran que las madres responsivas tenían más probabilidades de utilizar prácticas de alimentación basadas en estructura, establecimiento de límites y rutinas de alimentación consistentes. 
Tabla 4. Comparación intergrupo de las prácticas maternas de alimentación de acuerdo con la percepción materna del peso de los hijos y señales de hambre y saciedad

\begin{tabular}{|c|c|c|c|c|c|c|c|c|c|c|c|}
\hline \multirow[t]{3}{*}{ Grupo } & \multirow[t]{3}{*}{ Prácticas de alimentación } & \multicolumn{5}{|c|}{$\begin{array}{l}\text { Percepción materna } \\
\text { del peso del hijo }\end{array}$} & \multicolumn{5}{|c|}{$\begin{array}{l}\text { Percepción materna de } \\
\text { señales hambre-saciedad }\end{array}$} \\
\hline & & \multicolumn{2}{|c|}{ Adecuada } & \multicolumn{2}{|c|}{$\begin{array}{l}\text { Subestima o } \\
\text { sobrestima }\end{array}$} & \multirow[t]{2}{*}{ p } & \multicolumn{2}{|c|}{ Alta } & \multicolumn{2}{|c|}{$\begin{array}{l}\text { Pobre o } \\
\text { moderada }\end{array}$} & \multirow[t]{2}{*}{ p } \\
\hline & & $n$ & $\%$ & $\mathrm{n}$ & $\%$ & & $\mathrm{n}$ & $\%$ & $n$ & $\%$ & \\
\hline \multirow[t]{2}{*}{$\mathrm{GC}$} & Lactancia materna exclusiva & 3 & 10.00 & 11 & 36.70 & \multirow[t]{2}{*}{$0.426^{c}$} & 0 & 0.00 & 14 & 46.70 & \multirow[t]{2}{*}{0.533} \\
\hline & Alimentación con biberón & 5 & 16.60 & 11 & 36.70 & & 1 & 3.30 & 15 & 50.00 & \\
\hline \multirow[t]{2}{*}{$\mathrm{Gl}$} & Lactancia materna exclusiva & 19 & 63.34 & 6 & 20.00 & \multirow[t]{2}{*}{$0.232^{c}$} & 25 & 83.30 & 0 & 0.00 & \multirow[t]{2}{*}{0.009} \\
\hline & Alimentación con biberón & 4 & 13.30 & 1 & 3.40 & & 0 & 0.00 & 5 & 16.70 & \\
\hline
\end{tabular}

$\mathrm{GC}=$ grupo control, $\mathrm{Gl}=$ grupo intervención. ${ }^{\mathrm{c} P r u e b a ~ e x a c t a ~ d e ~ F i s h e r . ~}$

Finalmente, el peso del hijo al nacer no mostró diferencias significativas en ambos grupos; sin embargo, a los cuatro meses de edad se observaron más lactantes del Gl con peso adecuado y sin sobrepeso en relación con los del GC. Por lo tanto, proporcionar herramientas cognitivas a las futuras madres durante el embarazo puede incidir en conductas promotoras de la salud para ellas y sus hijos.

Esta es la primera vez que se estudian tres conceptos cognitivos en conjunto en futuras madres mexicanas para valorar su efecto en el estado nutricional del hijo lactante, que parecen ser prometedores para el control del peso del hijo posterior a su nacimiento. Sin embargo, se requieren más investigaciones en diferentes contextos socioeconómicos, con muestras representativas y por periodos más largos de seguimiento, para confirmar los resultados aquí expuestos.

\section{Conclusiones}

Fue posible determinar que la intervención educativa prenatal tuvo efecto sobre el estado nutricional de los lactantes, en quienes la obesidad ya es un problema de salud en México. Esta investigación muestra evidencia preliminar de la importancia del estudio de las cogniciones maternas en mujeres embarazadas para mejorar las condiciones de nutrición en los niños en la primera infancia.

\section{Agradecimientos}

Al Programa de Fomento y Apoyo a Proyectos de Investigación, PROFAPI 2015, por el financiamiento asignado para la presente investigación.

\section{Conflicto de intereses}

\section{Ninguno.}

\section{Financiamiento}

Se recibió financiamiento del Programa de Fomento y Apoyo a Proyectos de Investigación, PROFAPI 2015, de la Universidad Autónoma de Sinaloa.

\section{Responsabilidades éticas}

Protección de personas y animales. Los autores declaran que los procedimientos seguidos se conformaron a las normas éticas del comité de experimentación humana responsable y de acuerdo con la Asociación Médica Mundial y la Declaración de Helsinki.

Confidencialidad de los datos. Los autores declaran que siguieron los protocolos de su centro de trabajo sobre la publicación de datos de pacientes.

Derecho a la privacidad y consentimiento informado. Los autores obtuvieron el consentimiento informado de los pacientes o sujetos referidos en el artículo. Este documento obra en poder del autor de correspondencia.

\section{Bibliografía}

1. Organización Mundial de la Salud [Internet]. Suiza: Datos y cifras de obesidad infantil 2019; 2020.

2. Rivera JA, De Cossío T, Pedraza LS, Aburto TC, Sánchez TG, Martorell R. Childhood and adolescent overweight and obesity in Latin America: a systematic review. Lancet Diabetes Endocrinol. 2014:2:321-332.

3. Sanders RH, Han A, Baker JS, Cobley S. Childhood obesity and its physical and psychological co-morbidities: a systematic review of Australian children and adolescents. Eur J Pediatr. 2015;174:715-746.

4. Simmonds M, Llewellyn A, Owen CG, Woolacott N. Predicting adult obesity from childhood obesity: a systematic review and meta-analysis. Obes Rev. 2016;17:95-107. 
5. Moreno-Villares J, Collado MC, Larqué E, Leis-Trabazo MR, Sáenz-de-Pipaon M, Moreno-Aznar LA. Los primeros 1000 días: una oportunidad para reducir la carga de las enfermedades no transmisibles. Nutr Hosp. 2019;36:218-232.

6. Haines J, Haycraft E, Lytle L, Nicklaus S, Kok FJ, Merdji M, et al. Nurturing Children's healthy eating: position statement. Appetite. 2019; 137:124-133.

7. Harrison M, Brodribb W, Davies PSW, Hepworth J. Impact of maternal infant weight perception on infant feeding and dietary intake. Matern Child Health J. 2018;22:1135-1145.

8. Paul IM, Savage JS, Anzman-Frasca S, Marini ME, Beiler JS, Hess LB, et al. Effect of a responsive parenting educational intervention on childhood weight outcomes at 3 years of age: the INSIGHT randomized clinical trial. JAMA. 2018;320:461-468.

9. Savage JS, Birch LL, Marini M, Anzman-Frasca S, Paul IM. Effect of the INSIGHT responsive parenting intervention on rapid infant weight gain and overweight status at age 1 year: a randomized clinical trial. JAMA Pediatr. 2016;170:742-749.

10. Kim SK, Park S, Oh J, Kim J, Ahn S. Interventions promoting exclusive breastfeeding up to six months after birth: A systematic review and meta-analysis of randomized controlled trial. Int J Nurs Stud. 2018;80:94-105

11. Mennella JA, Papas MA, Reiter AR, Stallings VA, Trabulsi JC. Early rapid weight gain among formula-fed infants: Impact of formula type and maternal feeding styles. Pediatr Obes. 2019;14:e12503.

12. Ramírez-Pastore L, Gotz S, Sequera VG, Riera J, Brunilde P, Vera N et al. Percepción materna del estado nutricional de sus hijos que acuden a un consultorio pediátrico, Asunción, 2018. Pediatr. 2018;45:217-222.

13. Kim LP, Mallo N. Maternal perceptions of self-weight and child weight may influence milk choice of participants in the Special Supplemental Nutrition Program for Women, Infants, and Children (WIC). J Obes. 2019;3654728.

14. Zaragoza-Cortés J, Trejo-Osti LE, Ocampo-Torres M. Impact of a complementary feeding intervention and mother's perceptions of child weight status in infants. Nutr Hosp. 2019;36:282-289.

15. Blake-Lamb TL, Locks LM, Perkins ME, Woo Baidal JA, Cheng ER, Taveras EM. Interventions for childhood obesity in the first 1,000 days a systematic review. Am J Prev Med. 2016; 50:780-789.
16. Trejo-Ortiz P, Ahumada-Saucedo J. Factores asociados con la percepción materna del peso corporal del hijo: una revisión sistemática. Santiago VLIR. 2017;222-233.

17. Manikam LL, Lingam R, Lever I, Alexander E, Chidi A, Milner Y, et al. Complementary feeding practices for South Asian young children living in high-income countries: a systematic review. Nutrients. 2018;10:1676.

18. Eisenberg CM, Sánchez-Romero LM, Rivera-Dommarco, JA, Holub CK, Arredondo EM, Elder JP, et al. Interventions to increase physical activity and healthy eating among overweight and obese children in Mexico. Salud Publica Mex. 2013;55:441-446.

19. World Medical Association. World Medical Association Declaration of Helsinki ethical principles for medical research involving human subjects. JAMA. 2013;310:2191-2194.

20. Aristizábal-Hoyos GP, Blanco-Borjas DM, Sánchez-Ramos A, Ostiguín-Meléndez RM. El modelo de promoción de la salud de Nola Pender. Una reflexión en torno a su comprensión. Enferm Univ. 2011;8:16-23.

21. Organización Mundial de la Salud [Internet]. Suiza: Dieta, ejercicio o ambos para prevenir el aumento excesivo de peso en el embarazo; 2020.

22. Organización Mundial de la Salud [Internet]. Suiza: Patrones de crecimiento infantil; 2020

23. Huges SO, Power TG. Parenting influences on appetite and weight. En: Lumeg JC, Fisher JO, editores. Pediatric food preferences and eating behaviors. Reino Unido: Academic Press; 2018.

24. Organización Mundial de la Salud [Internet]. Suiza: Indicadores para evaluar las prácticas de alimentación del lactante y del niño pequeño; 2020.

25. Flores-Peña Y, Aguado-Barrera ME, Cerda-Flores RM, Cortés-Gutiérrez El, Dávila-Rodríguez MI. Percepción materna del peso del hijo y de niños no emparentados menores de un año. Aten Primaria. 2016:48:579-585.

26. Lewkowitz AK, Raghuraman N, López JD, Macone GA, Cahill AG. Infant feeding practices and perceived optimal breastfeeding interventions among low-income women delivering at a baby-friendly hospital. Am J Perinatol. 2019;36:669-677.

27. Kassianos AP, Ward E, Rojas-Garcia A, Kurti A, Mitchell FC, Nostikasari D, et al. A systematic review and meta-analysis of interventions incorporating behavior change techniques to promote breastfeeding among postpartum women. Health Psychol Rev. 2019;13:344-372. 